\title{
NOMA Enhanced 5G Distributed Vehicle to Vehicle Communication for Connected Autonomous Vehicles
}

\author{
Zuoyin Tang ${ }^{1}$, Jianhua $\mathrm{He}^{2}$ \\ ${ }^{1}$ School of Engineering and Applied Science, Aston University, UK. \\ ${ }^{2}$ School of Computer Science and Electronic Engineering, University of Essex, UK.
}

\begin{abstract}
Connected autonomous vehicles (CAV) holds great potentials of improving road safety and efficiency. However ultra reliability and low latency vehicle to everything (V2X) communication service is required to fully unleash the potentials of CAV. In this paper we investigate distributed vehicle to vehicle (V2V) for CAV, which supports not only broadcast but also multicast/unicast communications. Power domain nonorthogonal multiple access (NOMA) is applied to deal with the CAV traffic patterns, which are different from those in the traditional connected vehicle applications. With NOMA the signals for long range broadcast with major power and signals for short range neighbors with small power can be superposed in one transmission. With the application of NOMA the channel load can be reduced and communication reliability and latency will be improved. The framework and operation of NOMA enhanced distributed V2V system are designed. Qualitative and quantitative benefits of the proposed scheme are analyzed. Simulation results show that the proposed scheme can achieve a gain of more than $80 \%$ on network capacity under the investigated scenarios, with large performance improvement in terms of communication throughput and reliability.
\end{abstract}

Index Terms-LTE, 5G, vehicle to vehicle (V2V), connected vehicles, connected autonomous vehicles (CAV), NOMA.

\section{INTRODUCTION}

Road safety and car pollution are two major global transportation issues, e.g., road accidents claim more than 1 million lives worldwide every year, and transport pollution contributes significantly to the global climate change and extreme weathers. Automotive manufacturers and IT companies are racing to bring autonomous vehicles onto roads to tackle the road transport challenges. However the autonomous vehicles alone can not provide the required safety for autonomous vehicles especially under adverse weather conditions, even they are equipped with increasingly powerful sensors (e.g., camera, radar and LiDAR) and computing resources. Connected vehicles is receiving revived interests from industry and academia, as it can work with autonomous vehicles to provide safer and more efficient transport. The integrated connected vehicles and autonomous vehicles, which is called connected autonomous vehicles (CAV), holds great potentials on building cooperative context awareness and cooperative driving to achieve the goals of zero road accidents [1].

As safety is the most important for autonomous driving and road transport, to ensure the autonomous vehicle safety and fully unleash the potentials of CAV, ultra reliability and low latency vehicle to everything (V2X) communication service will be needed. There are two mainstream V2X technologies, random channel access based IEEE 802.11p technology, and resource reservation based cellular vehicle to everything (cellular V2X). IEEE $802.11 \mathrm{p}$ has been specified for V2V and vehicles to roadside infrastructure (V2I) data exchange [2] [3]. Evolved version IEEE $802.11 \mathrm{bd}$ was specified recently for enhanced V2X performance [7]. 3GPP developed LTE specifications to support vehicle to everything (V2X) communications [4], [5], [9] and enhanced 5G V2X services for advanced CAV applications [6]. Compared to IEEE 802.11p, 3GPP V2X technologies can provide better performance, e.g., higher spectrum utilization with synchronized communication, better QoS in terms of latency and reliability with centralized network and/or resource management from eNodeBs. The infrastructure investment for $5 \mathrm{G}$ V2X applications can also be significantly reduced by reusing existing cellular system infrastructure.

In view of the CAV communication needs and the capabilities of the V2X technologies, we are motivated to investigate the 5G V2X technologies with focus on distributed V2V communications for CAV. For advanced CAV applications, the vehicles will have different traffic patterns in the traditional connected vehicles, which mainly transmit periodic safety data packets (with information such as position and driving status) by broadcast to build mutual driving context awareness. In addition to the general short broadcast data packet for long range coverage, the CAVs will also share more specific and richer information (such as detected objects and camera views) with close range vehicles for applications such as cooperative perception and cooperative driving, by the means of unicast, multicast or broadcast. To improve the $\mathrm{V} 2 \mathrm{~V}$ communication performance for $\mathrm{CAV}$, we apply power domain non-orthogonal multiple access (NOMA) to the $5 \mathrm{G}$ distributed $\mathrm{V} 2 \mathrm{~V}$, which is ideally fit for the CAV traffic patterns. With NOMA the signals for long range broadcast and short range neighbors can be supercomposed in one transmission. Application of NOMA can reduce the channel load, and communication reliability and latency will be improved. While the proposed idea can be extended for NOMA in other domains (such as code domain) and vehicle to infrastructure (V2I) for CAV.

The remaining of the paper is organized as follows. We introduce the background knowledge and related works in 


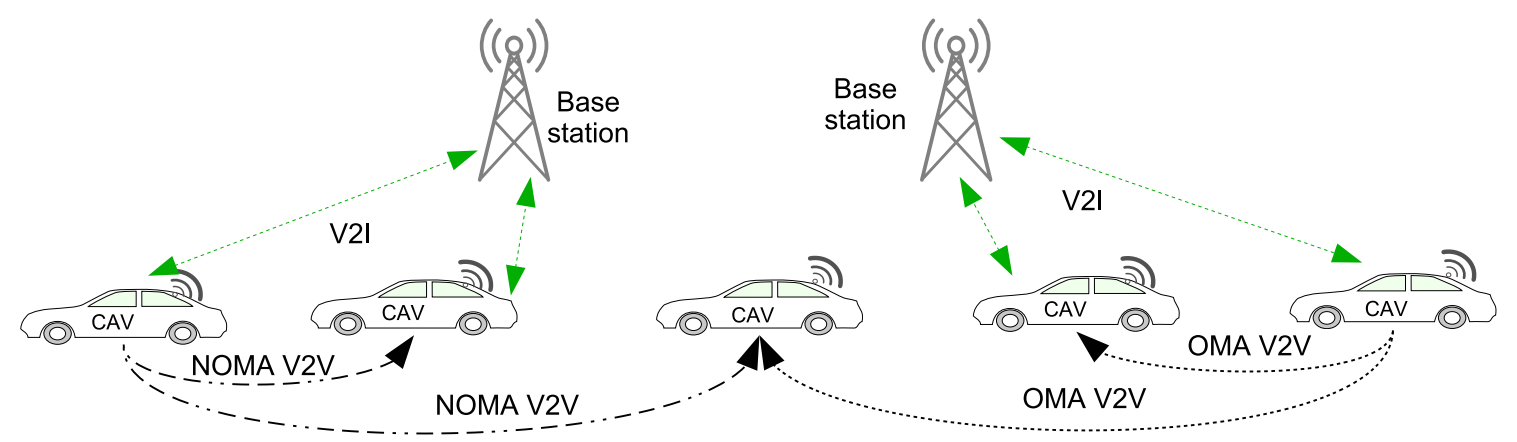

CAV: connected autonomous vehicles; V2V: vehicle to vehicle; V2I: vehicle to infrastructure

OMA: orthogonal multiple access; NOMA: non-orthogonal multiple access

Fig. 1. Illustration of CAV networks and V2V communications. In the OMA scheme normal periodic CAM broadcast messages and CRVM message are transmitted in separate data packets. In the NOMA scheme CAM and CRVM messages can be superposed in one data packet transmission.

Section II. The framework and operation of proposed NOMA enhanced distributed V2V system are presented in Section III. Preliminary results showing the potentials of the NOMA enhanced distributed V2V for CAVs are presented and discussed in Section IV. Our work demonstrates the large potentials of CA with better vehicle communication.

\section{RELATED WORKS}

\section{A. Evolution of $V 2 X$}

Support of V2X services was specified in the 3GPP Release 14 LTE standards, which can be provided over PC5 interface by sidelink transmissions [4], [5]. The V2X transmissions are controlled by centralized scheduling via the eNodeBs or distributed scheduling by the vehicles. Centralized broadcast scheduling has high broadcast reliability, but signalling overheads could be very high due to vehicle position update and resource allocation [8], [10]. Distributed scheduling with autonomous resource selection has higher scalability. The sidelink transmission for V2X was enhanced in 3GPP Release 15 , with new functions such as transmission diversity, carrier aggregation and higher quadrature amplitude modulation. The 3GPP Release 16 specifies $5 \mathrm{G}$ new radio (NR) based V2X support with further new functions, including 1) additional support for unicast and multicast, which are particular useful for CAV applications; 2) grant free transmissions which can improve latency; 3) enhanced channel sensing and resource selection; 4) QoS management schemes and congestion control [6]. V2V performance with collision avoidance (CA) and location based resource allocation enhancements have been reported for autonomous resource selection in [4] [9]. An enhanced collision avoidance scheme which uses data packets to disseminate the reservation for both schedule assignment (SA) and data resources was reported in [9], which provides better resource reservation and reduces collisions.

\section{B. NOMA in Vehicular Communications}

In an orthogonal multiple access (OMA) system, such as orthogonal frequency division multiple access (OFDMA), frequency-time resource is allocated exclusively to at most one user equipment (UE) simultaneously in the same cell. NOMA systems allow simultaneous allocation of the same frequency resource to multiple UEs in the same cell. Non-orthogonal resource allocation and signal reception can be achieved in power and code domains [11].

Due to the superior spectrum efficiency and massive connectivity NOMA has been widely studied recently for 5G systems [10], [11]. As NOMA can improve communication latency and channel access capacity, its application to $\mathrm{V} 2 \mathrm{X}$ received increasing interests [10]. It is noted that most of the existing research works on NOMA application to $\mathrm{V} 2 \mathrm{X}$ are focused on the V2I links, where resource allocation is centralized. And the performance evaluation is mainly based on theoretic analysis. In this paper we focus on the $\mathrm{V} 2 \mathrm{~V}$ links where resources are allocated in a distributed way by the vehicles. And the performance evaluation is conducted with a discrete events driven simulator.

\section{System ARChitecture And Assumptions}

In this paper we consider autonomous $\mathrm{V} 2 \mathrm{~V}$ resource selection for CAV communications. There are $N_{v}$ connected autonomous vehicles in the network. Vehicles broadcasts periodic cooperative awareness messages (CAM) data packets to their neighbors. CAM are assumed to be generated with a period of $T_{p}$ ms. The messages carry the host vehicle's information such as speed, heading, brake status as well as security overhead, which are used to build cooperative awareness and support cooperative safety applications. Each data packet is assumed to have an overall length of 190 bytes. The latency requirement for data packets is denoted by $T_{d}$ (set to 100 ) in $\mathrm{ms}$.

The connected vehicles are all equipped with one transmission antenna and operate in half-duplex mode. The 3GPP 5G uplink PHY and MAC specifications are used for broadcast communication. Orthogonal frequency division multiplexing (OFDM) is used at the PHY layer and single carrier frequency division multiple access (SC-FDMA) is used at the MAC layer. Transmissions are synchronized with $10 \mathrm{~ms}$ frames and $1 \mathrm{~ms}$ subframes. Each subframe has two time slots of 0.5 ms. Spectrum frequency is divided into subcarriers of 15 $\mathrm{KHz}$. The minimal unit of resource block (RB) for resource 
allocation has 1 time slot and 12 consecutive subcarriers in frequency. The resource blocks are organized to fixed size SA and data subchannels for transmission of SA and data packets, respectively. All data packet transmissions are scheduled by SA. The numbers of RBs included in SA and data subchannels depend on the size of SA and data packets, and the used modulation and coding schemes (MCS).

In this paper, we assume SA subchannel includes $1 \mathrm{RB}$ and data subchannel includes $14 \mathrm{RB}$. Each transmission resource for SA and data packets has one SA and data subchannel over one subframe, respectively Let $N_{s a}$ and $N_{d t}$ denote the number of SA and data transmission resources in one subframe, respectively, which can be determined according to the system bandwidth, MCS and the packet sizes [4] [12]. An example of frame structure and resource organization is shown in Fig. 2, where the system spectrum resource is divided to SA and data resource pools, and there are 4 SA resources and 2 data resources in one subframe, respectively.

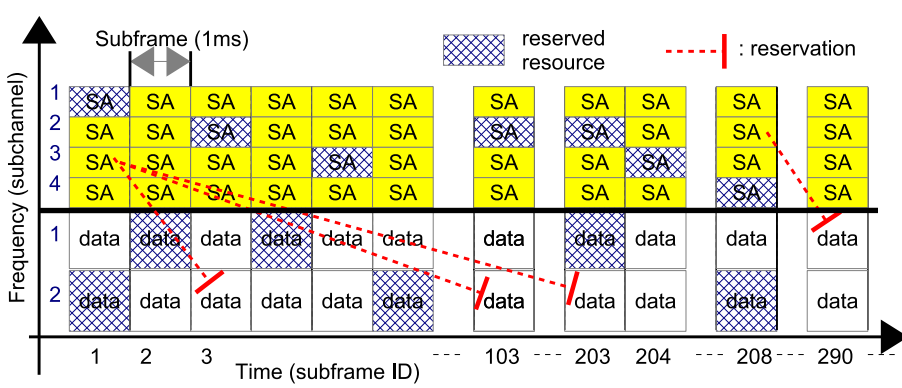

Fig. 2. Illustration of frame structure and resource organization, with 4 SA resources and 2 data resources in one subframe.

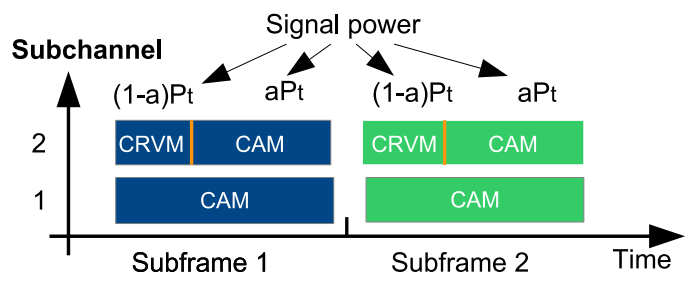

Fig. 3. Signal superposition. Long range CAM and short range CRVM messages are superposed in subchannel 2. $\alpha$ is the proportion of transmit power $P_{t}$ allocated to CAM.

\section{NOMA ENHANCED V2V COMMUNICATIONS FOR CAV}

\section{A. Basic Collision Avoidance Algorithm}

In the basic collision avoidance (CA) algorithm, there is a resource selection and resource reservation procedure, which is needed for data transmissions. Resource reservation is made by vehicles over SA subchannels. Vehicles keep monitor the channel, receiving SA and data packets from neighbor vehicles. The time and frequency location of the reserved resources for data transmissions can be extracted from successfully decoded SAs. The SA and data packets from each vehicle are transmitted in different subframes. Receiving vehicles set the resources selected by the received SAs as reserved.

When a vehicle has data packets to transmit, it can only select unreserved resources for them. Multiple resources for CAM can be selected and reserved in one process. Then a SA message is scheduled and sent over the SA subchannel to announce the reservation of the data resources. Let $T_{\text {curr }}$ denote the time (sequence number of subframe) when the scheduling process starts. The SA is scheduled to a SA resource which is selected randomly from the unreserved SA resources from the unreserved SA resources within the time window $\left[T_{\text {curr }}+T_{\text {sa,low }}, T_{\text {curr }}+T_{\text {sa,up }}\right] . T_{\text {sa,low }}$ and $T_{\text {sa,up }}$ are configurable lower and upper limits of the SA resource selection window, respectively. $T_{\text {sa,low }}$ and $T_{\text {sa,up }}$ are set to 2 and 6 in this paper with the consideration of packet preparation and a tradeoff in the randomness and transmission latency. If in the process of scheduling a SA there is no unreserved SA resource in the corresponding time window, the next SA resource selection window is used to schedule this SA. Let $N_{\text {sch }}$ denote the number of SA transmissions that are successfully scheduled.

For each scheduling process, the number of data resources to be reserved (denoted by $N_{r s v}$ ) for CAM is randomly selected from $\left[N_{\text {low }}, N_{\text {up }}\right] . N_{\text {low }}$ and $N_{u p}$ are configurable parameters, which are set to 5 and 10, respectively. Once the first CAM data packet is scheduled, the remaining data packets are scheduled similarly.

\section{B. NOMA Enhanced V2V Operation}

In the CAV systems, we assume that in addition to the traditional periodic CAM broadcast messages there are new close range vehicles messages (CRVM), e.g., messages exchanged with immediately front vehicles for cooperative perception. There can be single or multiple destinations for a CRVM. The expected communication range of CAM is typically 250 meters, while the one for CRVM can be less than 50 meters. Due to the short communication range of CRVM they could be superposed to the CAM by NOMA to reduce the traffic loads over the control and data channels.

In the NOMA enhanced V2V system, the transmission of the periodic CAM follows the CA algorithm for resource selection and reservation. We assume that CRVM are generated on demands (e.g., on the requests from close range neighbor vehicles). Suppose a vehicle knows it has CRV messages to transmit. It will check if the channel condition is favourable for a superposition of its CAM and CRVM through NOMA. If the condition allows, then the vehicle announces the composition of the CAM and CRVM in the SA packets, with a specific flag indicating the use of NOMA and a specified receiver group (e.g., the vehicles within a range of 50 meters of this transmitting vehicle). Otherwise, the CAM and CRVM will go through separate resource reservation procedures and are transmitted in separate data packets.

Consider the cases of a CAM and a CRVM being sent through one data packet by NOMA. 
1) Trainsmission: The transmit vehicle allocate a larger proportion (say 90\%) of transmit power to the long range broadcast CAM, and the remaining transmit power to the short range CRVM. The sum transmit power can be kept the same as or a little bit larger than that set for the normal CAM data packets. An illustration of the signal superposition is illustrated in Fig.3, where OMA signal is transmitted over subchannel 1 and NOMA signal is transmitted over subchannel 2 .

2) Reception: In the receivers' side, they decode the broadcast CAM as normal. For the target receivers of the CRVM which are included the specified receiver group in the SA packets, they will apply successive interference cancellation technique to decode both CAM and CRVM as follows. They first decode and process the broadcast CAM, then cancel the CAM signal, and decode and process the CRVM.

In the communication process the target receivers of CRVM may miss a few CRVM, which is not a big issue, as it could be mitigated by retransmission of CRVM with the CAM as needed. The proposed scheme is compatible with the legacy distributed $\mathrm{V} 2 \mathrm{~V}$ scheme. The legacy receivers can simply ignore the additional flag and information carried in the SAs and the superposed CRVM in the data packets.

\section{Signal to Interference and Noise Ratio}

For simplicity, we assume that the total transmit power for all data packets is fixed at $P_{\mathrm{t}}$. Consider a general vehicle $u$ for a data packet, which is away from the transmit vehicle with a distance $d$. Then the received power can be computed by:

$$
\mathcal{P}_{r}=P_{\mathrm{t}} G_{\mathrm{PL}}(d) G_{\mathrm{A}} \psi \phi,
$$

where $G_{\mathrm{PL}}(d)$ denotes the path loss between the two vehicles, $G_{\mathrm{A}}$ is the antenna gain, $\psi$ denotes the shadow fading and $\phi_{r}$ denotes small scale fast fading [11].

In the case of OMA packet transmission, each data packet includes only one message (CAM or CRVM). Let $\gamma_{d}^{\mathrm{s}}$ denote the signal to interference plus noise ratio (SINR) at distance $d$, which can be computed as:

$$
\gamma_{d}^{\mathrm{s}}=\frac{\mathcal{P}_{r}}{\mathcal{P}_{i}+\mathcal{P}_{n}} .
$$

where $\mathcal{P}_{i}$ and $\mathcal{P}_{n}$ denotes the interference from other transmitting vehicles and noise power, respective.

In the NOMA packet transmission, a data packet carries both CAM and CRVM messages. The signals for CAM and CRVM messages are superimposed, with transmit power $\alpha P_{\mathrm{t}}$ and $(1-\alpha) P_{\mathrm{t}}$ allocated to CAM and CRVM messages, respectively. Power allocation coefficient $\alpha$ is configuration and should be larger than 0.5 .

If a vehicle is not included in the specified receiver group for a received NOMA data packet, it can decode the CAM message from the data packet directly without SIC; otherwise it will apply SIC to decode the CAM message first and then the CRVM message. Let $\gamma_{a}^{\mathrm{m}}(d)$ and $\gamma_{v}^{\mathrm{m}}(d)$ denote the SINR for the CAM and CRAM messages at a receive vehicle with distance $d$, respectively. We can compute $\gamma_{a}^{\mathrm{m}}(d)$ by:

$$
\gamma_{a}^{\mathrm{m}}(d)=\frac{\alpha \mathcal{P}_{r}}{(1-\alpha) \mathcal{P}_{r}+\mathcal{P}_{i}+\mathcal{P}_{n}} .
$$

And CRVM SINR $\gamma_{v}^{\mathrm{m}}(d)$ can be computed by:

$$
\gamma_{v}^{\mathrm{m}}(d)=\frac{(1-\alpha) \mathcal{P}_{r}}{\mathcal{P}_{i}+\mathcal{P}_{n}} .
$$

The above formulae will be used in the network simulations and can also be used for analytical performance evaluation.

\section{Performance Evaluation of NOMA Enhanced DISTRIBUTED V2V}

\section{A. Qualitative Performance Analysis}

Qualitatively speaking, the proposed scheme can improve the distributed $\mathrm{V} 2 \mathrm{~V}$ performance for CAV from at least two aspects: 1) As CRVM can be transmitted with periodic CAM, they don't need separate SA packets to reserve resources. As SA resource reservation is a major bottleneck for distributed $\mathrm{V} 2 \mathrm{~V}$, the proposed scheme could significantly reduce SA subchannel congestion and collisions of data packets. 2) The superposition of CAM and CRVM can improve the spectrum utilization and reduce the collision of packets and latency, which will greatly improve the reliability and latency of distributed V2V for CAV applications. Quantitively, if we assume that CRVM are generated with the same frequency and the same size as those of CAM, under saturated traffic conditions, the throughput of the distributed V2V could be improved by at least $100 \%$. More evaluation results of the proposed scheme will be added later.

\section{B. Simulation Results}

We developed a system-level simulator to evaluate the proposed CA algorithm for distributed LTE V2V broadcast under highway scenarios. For each simulation 200 seconds (200 thousands subframes) are simulated. And 10 simulations are run to obtain an averaged result. In the normal distribute $\mathrm{V} 2 \mathrm{~V}$ scheme vehicles select unoccupied data resources and update resource reservation according to the received SAs.

Main simulation parameters are set according to [4] and [9], which are shown in Table. I. Vehicle density varies from 150 to 210 vehicles per $\mathrm{km}$. Vehicles are randomly dropped to the road with uniform distribution. Form simplicity, data packet generation periods for both CAM and CRVM are $100 \mathrm{~ms}$.

TABLE I

SYSTEM PARAMETERS.

\begin{tabular}{l|l|l|l}
\hline Parameter & Value & Parameter & Value \\
\hline Carrier freq. & $6 \mathrm{GHz}$ & Bandwidth & $10 \mathrm{MHz}$ \\
\hline SA resources & $5 / \mathrm{subframe}$ & data resources & $2 /$ subframe \\
\hline Antenna height & $1.5 \mathrm{~m}$ & Antenna gain & $3 \mathrm{dBi}$ \\
\hline TX Antenna & 1 & RX Antenna & 2 \\
\hline Tx power & $23 \mathrm{dBm}$ & Noise figure & $9 \mathrm{~dB}$ \\
\hline Path loss & WINNER+B1 [4] & Fast fading & ITU-R UMi [13] \\
\hline Shadowing & Log-normal & Std. dev. & $3 \mathrm{~dB}$ \\
\hline Data MCS & QPSK, Turbo 1/2 & Road length & $2000 \mathrm{~m}$ \\
\hline Number of lanes & 6 & Lane width & $4 \mathrm{~m}$ \\
\hline Vehicle speed & $70 \mathrm{~km} / \mathrm{h}$ & Speed std. dev. & $5 \mathrm{~km} / \mathrm{h}$ \\
\hline
\end{tabular}




\section{Simulation Results}

The performance metrics of interest include packet reception ratio (PRR) and network capacity. For a transmitted packet with ID $n$, the average PRR of this packet over a given range is calculated as the ratio of the number of vehicles successfully receiving the packet (denoted by $V_{s u c, n}$ ) to the number of vehicles located in the range (denoted by $V_{n}$ ). Suppose that $N_{t x}$ packets are transmitted in a simulation. Then the network wide average PRR for that range is computed by $\sum_{n=1}^{N_{t x}} V_{s u c, n} / \sum_{n=1}^{N_{t x}} V_{n}$. To determine the network capacity, we set the target packet reception distance for the CAM data packets to $320 \mathrm{~m}$, and the target average PRR for CAM messages in the range $(300,320) \mathrm{m}$ from transmitters to $90 \%$. The largest vehicle density with which the target CAM PRR can be achieved in the range $(300,320)$ is defined as the network capacity.

We compare the average PRR of the CAM messages with the normal distributed V2V communication scheme (labelled "OMA V2V") and the NOMA enhanced scheme (labelled "NOMA V2V") at different transmitter ranges. A random number of data resources reserved in the range $[5,10]$ per SA. Representative CAM message PRR results versus transmitter distance are presented in Fig.4. The vehicle density is fixed as 200 vehicles $/ \mathrm{km}$. The link layer communication performance of data PRR against SNR presented in [13] is used in simulations. The link model PRR performance (labelled "Link Model") is also included in the figure, which assumes no SA and data packet collision, therefore representing the upper limits of the broadcast schemes. As the PRR for CRVM messages is very close to that of the CAM messages, CRVM PRR results are not presented.

It can be observed that the NOMA V2V scheme has much better performance than the OMA V2V scheme. For example, at the transmit distance of 300 meters, the PRR with NOMA $\mathrm{V} 2 \mathrm{~V}$ is about 0.86 while it is 0.65 for OMA V2V. Following the definition of the network capacity (achieving the target PRR of $90 \%$ in the transmit range of $(300,320)$, according to the simulation results the NOMA V2V scheme has a network capacity of 165 vehicles/ $\mathrm{km}$, while the OMA V2V scheme has only a network capacity of 90 vehicles $/ \mathrm{km}$. The gain on network capacity by the proposed NOMA V2V scheme is $83.3 \%$. The results demonstrate that the NOMA scheme can significantly improve the performance of distribute $\mathrm{V} 2 \mathrm{~V}$ communication in terms of reliability, throughput, latency and network capacity.

\section{CONCLUSION}

In this paper we proposed a NOMA enhanced distributed $\mathrm{V} 2 \mathrm{~V}$ scheme for $\mathrm{CAV}$, for advanced cooperative perception and driving safety applications. Power domain NOMA is applied to deal with the CAV traffic patterns, with signals for long range broadcast with major power and signals for short range neighbors with small power being superposed in one transmission. With the application of NOMA the traffic loads on both control and data channels can be reduced, and communication reliability and latency will be improved.

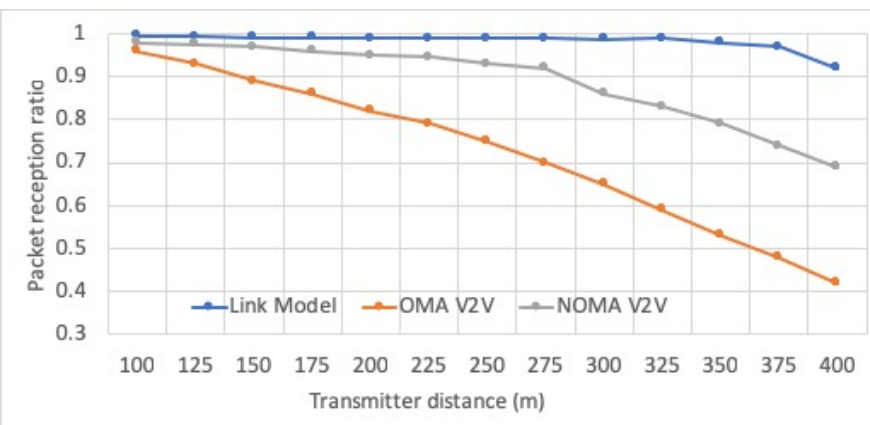

Fig. 4. Average PRR of the CAM messages versus transmitter distance for different schemes. Vehicle density is 200 vehicles $/ \mathrm{km}$.

Simulation results show that the proposed scheme achieved more than $80 \%$ network capacity under the investigated scenarios than the OMA scheme, And communication throughput and reliability are also largely improved. In future adaptive power allocation, code domain NOMA based distributed V2V schemes and the impact of the improved $\mathrm{V} 2 \mathrm{~V}$ communication on CAV applications will be investigated.

\section{ACKNOWLEDGEMENT}

This project has received funding from the European Union's Horizon 2020 research and innovation programme under the Marie Skłodowska-Curie grant agreement No 824019.

\section{REFERENCES}

[1] J. He, et al, 'Cooperative Connected Autonomous Vehicles (CAV): Research, Applications and Challenges,' IEEE 27th International Conference on Network Protocols, 2019.

[2] 'TS 102 687: intelligent transport systems (ITS); decentralized congestion control mechanisms for intelligent transport systems operating in the $5 \mathrm{GHz}$ range; access layer part', ETSI, July 2011.

[3] J. He, H. Chen, et al, 'Adaptive congestion control for DSRC vehicle networks', IEEE Comm. Lett., Feb. 2010, 14, (2), p.127-129.

[4] "Study on LTE-based V2X services", 3GPP TR 36.885, V14.0.0, June 2016.

[5] S. Chen, et al, 'Vehicle-to-Everything (v2x) services supported by LTEbased systems and 5G,' IEEE Comm. Std. Mag., June 2017, 1, (2), p.70 - 76.

[6] S. Lien, et al, '3GPP NR Sidelink Transmissions Toward 5G V2X,' IEEE Access, Vol.8, p.35368-35372, Feb. 2020.

[7] G. Naik, B. Choudhury, J. Park, 'IEEE 802.11bd \& 5G NR V2X: Evolution of Radio Access Technologies for V2X Communications,' IEEE Access, Vol.7, p.70169-70185, June 2019.

[8] X. Zhang, et al, 'Research on overlay D2D resource scheduling algorithms for V2V broadcast service', IEEE VTC, Sept. 2016.

[9] J. He, Z. Tang, Z. Fan, J. Zhang, 'Enhanced collision avoidance for distributed LTE vehicle to vehicle broadcast communications,' IEEE Communications Letters, Vol.22, No.3, p. 630-633, Jan. 2018.

[10] B. Di, et al, 'Non-orthogonal multiple access for high-reliable and lowlatency V2X communications in 5G systems', IEEE JSAC, July 2017.

[11] J. He, et al, 'Design and Optimization of Scheduling and NonOrthogonal Multiple Access Algorithms with Imperfect Channel State Information,' IEEE Trans. Vehicular Technologies, Vol.67, No.11, p. 10800-10814, Sept. 2018.

[12] G. Cecchini, 'LTEV2Vsim: An LTE-V2V simulator for the investigation of resource allocation for cooperative awareness', IEEE Conf. Models and Technologies for ITS, June 2017.

[13] "Vehicle to Vehicle (V2V) services based on LTE sidelink; User Equipment (UE) radio transmission and reception", 3GPP TR 36.785, V14.0.0, Oct. 2016 